\title{
TWO CASES OF BULLOUS PEMPHIGOID INDUCED BY VILDAGLIPTIN
}

\author{
Authors: Asli Dogruk Unall ${ }^{1}$, Ozlem Tarcin ${ }^{1}$, Gulsen Tukenmez Demirci' ${ }^{2}$, Ebru Demiralay ${ }^{3}$, \\ Ayse Tulin Mansur ${ }^{2}$, Semsi Yildiz², Nilgun Guvener Demirag ${ }^{1}$ \\ 1Baskent Üniversity Istanbul Hospital, Department of Endocrinology \\ 2Baskent University Istanbul Hospital, Department of Dermatology \\ ${ }^{3}$ Baskent University Istanbul Hospital, Department of Pathology
}

Background: Bullous pemphigoid is an autoimmune blistering disease that commonly arise in elderly with increased risk for mortality and morbidity. The etiology of this disease is not entirely clear, although a few cases have been described with dipeptidyl peptidase IV inhibitors.

Case 1: A 81-year old man presented with bullous pemphigoid after 28-month treatment with repaglinide, vildagliptin and metformin. The patient's condition didn't improve with local treatment with clobetasol. The patient was started on methylprednisolone and azathiopurine but skin lesions sustained. Remission was achieved only after withdrawal of gliptin.

Case 2: A 64-year old man treated with insulin glargine, insulin aspart and vildagliptin for 12 months presented with bullous pemphigoid on skin and laryngeal mucosa. After discontinuation of vildagliptin skin lesions were resolved but laryngeal involvement continued. Mucosal lesions were resolved after methyprednisolone and azathiopurine treatment.

Discussion: Although the exact pathogenetic mechanism of BP has not been known yet, it has been believed that modified immune responses might be the cause. In the literature, there have been few cases descibed with gliptins. Here we present two cases of BP induced by vildagliptin. According to Narenjo Advers Drug Reaction Probability Scale, it may be probable to be due to drug and the score was estimated as 7. BP is very rarely seen disease but its prevelance increases after age of 70 . Generally the cases in the literature had been reported after 2-13 months of gliptins. In our first case the eruption begun after 28 months of vildagliptin and in the second case rarely seen mucosal involvement was diagnosed.

Conclusion: These reports support the risk of bullous pemphigoid in patients exposed to gliptins. The difference of our cases from the literature is mucosal involvement and remission of our cases with immunosupressive agents and withdrawal of vildagliptin. We should advice the patients about the skin eruptions when we intend to begin treatment with gliptins.

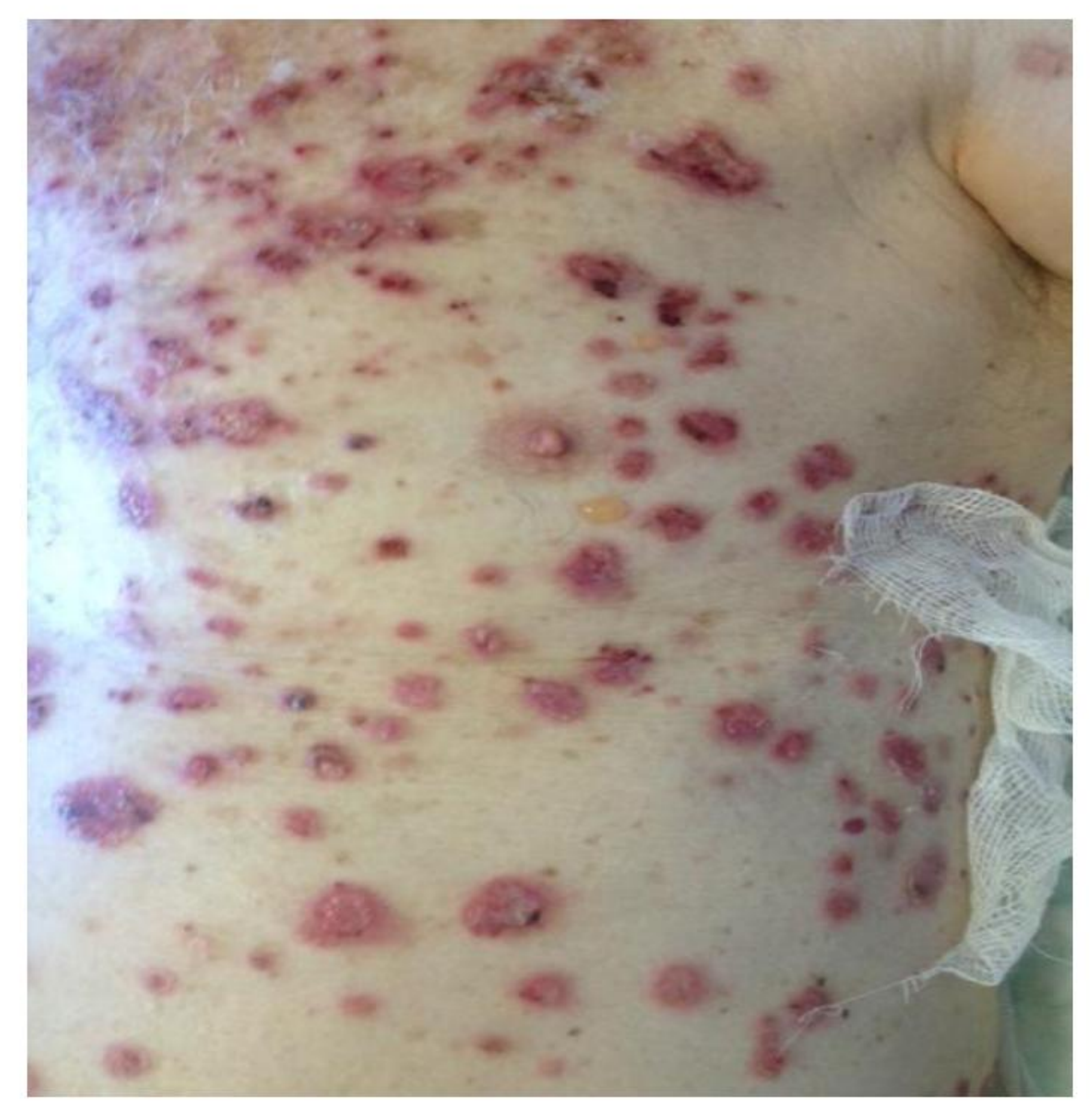

Figure-1:Skin eruptins in BP patient

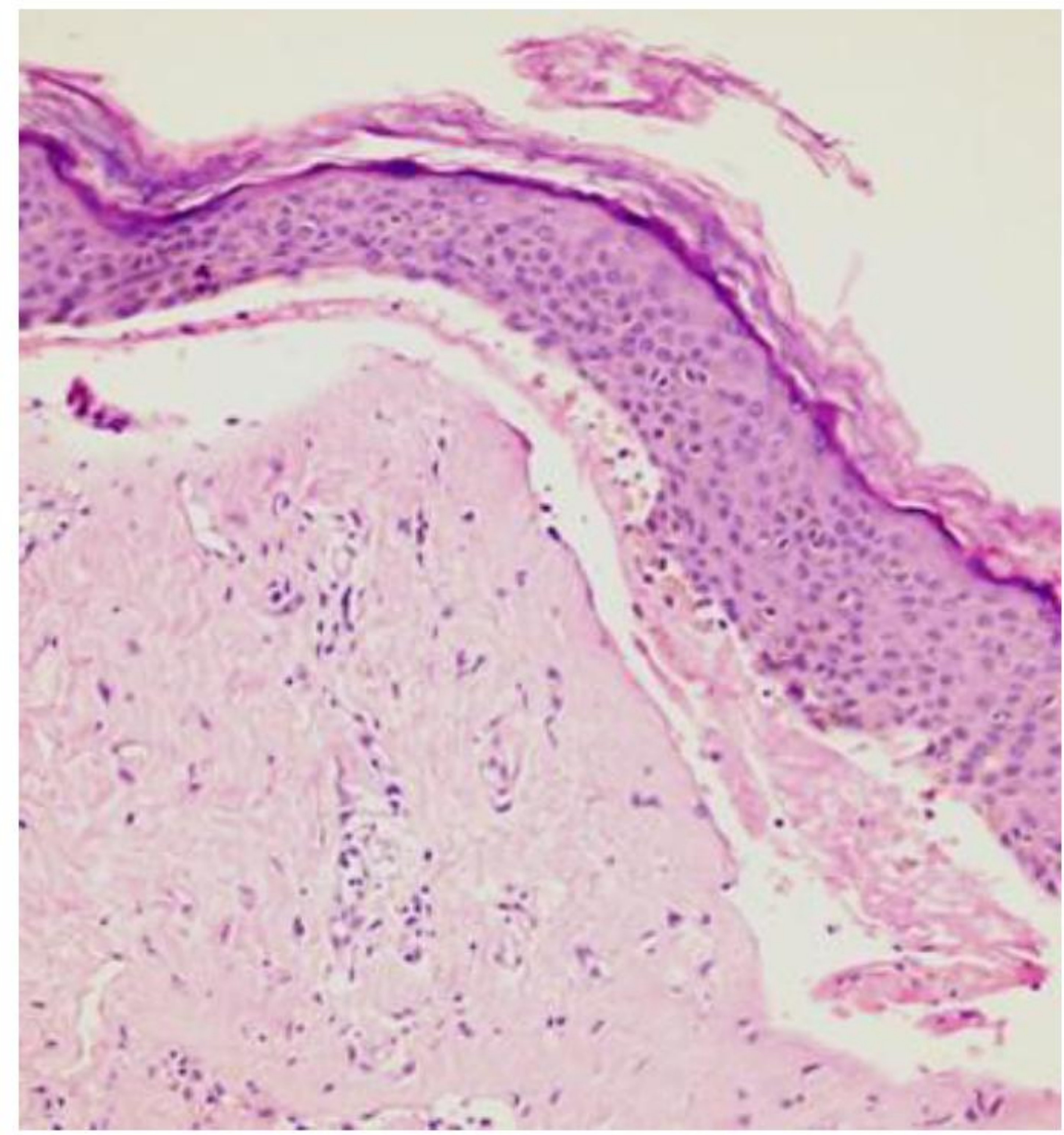

Figure-2: H\&E staining of skin biopsy from BP patient 